\title{
Interactions of Conjugated Linoleic Acid and Lipoic Acid on Insulin Action in the Obese Zucker Rat
}

\author{
Mary K. Teachey, Zachary C. Taylor, Thomas Maier, Vitoon Saengsirisuwan, Julie A. Sloniger, Stephan Jacob, \\ Martin J. Klatt, Arne Ptock, Klaus Kraemer, Oliver Hasselwander, and Erik J. Henriksen
}

\begin{abstract}
The fatty acid conjugated linoleic acid (CLA) and the antioxidant $R-(+)-\alpha$-lipoic acid (R-ALA) individually enhance glucose tolerance and insulin action on skeletal muscle glucose transport in the insulin-resistant obese Zucker rat. To date, no study has assessed the potential interactions between these 2 interventions in treating insulin resistance. The present study was designed to determine whether chronic treatment with CLA and R-ALA in combination would enhance skeletal muscle glucose transport to a greater extent than either intervention individually. CLA, R-ALA, or a combination treatment of R-ALA and CLA were administered to female obese Zucker rats for 20 days at low or high doses. Whereas low-dose R-ALA (10 mg/kg body weight) alone did not alter muscle glucose transport, low-dose CLA $(0.3 \mathrm{~g} / \mathrm{kg})$ induced a significant increase $(38 \%, P<$ .05 ) in insulin-mediated glucose transport in epitrochlearis, but not in soleus. Low-dose combination therapy brought about the greatest enhancement of insulin-mediated glucose transport in epitrochlearis $(77 \%)$ and soleus $(54 \%)$, with the latter effect being associated with a $50 \%$ reduction in protein carbonyls (an index of tissue oxidative stress) and a $33 \%$ diminution in muscle triglycerides. High-dose treatments with CLA $(1.5 \mathrm{~g} / \mathrm{kg})$, R-ALA $(50 \mathrm{mg} / \mathrm{kg})$, and the combination of CLA and R-ALA elicited increases in insulin-mediated glucose transport in epitrochlearis (57\%, 58\%, and $77 \%)$ and soleus (32\%, 35\%, and $54 \%)$. However, whereas the individual high-dose treatments with CLA and R-ALA reduced protein carbonyls $(63 \%$ and $49 \%)$ and triglycerides $(29 \%$ and $28 \%)$ in soleus, no further reductions were observed with the high-dose combination treatment groups. These findings support a significant interaction between low doses of CLA and R-ALA for enhancement of insulin action on skeletal muscle glucose transport, possibly via reductions in muscle oxidative stress and in lipid storage.
\end{abstract}

(c) 2003 Elsevier Inc. All rights reserved.

NSULIN RESISTANCE of skeletal muscle glucose disposal is a primary defect leading to the development of glucose intolerance and type 2 diabetes. ${ }^{1,2}$ This insulin resistance and the accompanying hyperinsulinemia are closely associated with a number of atherogenic risk factors, including hypertension, dyslipidemia, and central obesity, in a condition described as "syndrome $X " 3$ or the "insulin resistance syndrome." 4 The implementation of effective strategies for reducing insulin resistance of skeletal muscle glucose transport is therefore important in the prevention and treatment of type 2 diabetes. Although physical activity and fat mass loss are the preferred interventions for improving insulin action under conditions of insulin resistance, ${ }^{5}$ these interventions often fail because of a lack of long-term subject compliance. As a result, pharmaceutical and nutriceutical treatments have become alternative therapies for ameliorating insulin resistance.

The fatty acid conjugated linoleic acid (CLA) and the antioxidant $\alpha$-lipoic acid (ALA) have each been studied individually to assess their ability to improve glucose tolerance and insulin action. CLA is a naturally occurring compound found in the meat and milk of ruminating animals. In addition to its actions against cancer, ${ }^{6}$ CLA has been shown to also have anti-obesity ${ }^{7}$ and antidiabetic ${ }^{8,9}$ effects and to mediate a repartitioning of the fat-to-lean mass ratio. ${ }^{10-12}$ CLA is thought to act through the peroxisome proliferator-activated receptor- $\gamma$ (PPAR- $\gamma$ ) to elicit the beneficial effects in animal models of diabetes. ${ }^{8}$ In addition, CLA displays antioxidant properties, ${ }^{13}$ and its ability to neutralize reactive oxygen species may be responsible for at least some of its beneficial metabolic effects.

The R-(+)-enantiomer of ALA (R-ALA) is a well-described biological antioxidant. ${ }^{14}$ As a free radical scavenger, R-ALA decreases levels of oxidative stress and protein carbonylation in skeletal muscle, liver, and myocardium of the lean and obese Zucker rats. ${ }^{15,16}$ Exogenous treatment with R-ALA has also been shown previously to improve insulin-stimulated whole body and skeletal muscle glucose disposal in the insulin-resis- tant obese Zucker rat ${ }^{15,17-19}$ and treatment with a racemic mixture of ALA enhances whole-body glucose disposal in investigations of type 2 diabetic human subjects. ${ }^{20-23}$

Because evidence to date indicates that the effects of CLA and R-ALA are mediated through different cellular mechanisms (the former through activation of PPARs and the latter via a reduction in oxidative stress), it was hypothesized that when CLA and R-ALA are used in combination, there would be at least an additive interaction in improving insulin-stimulated glucose transport in skeletal muscle of insulin resistant animals and humans. In this context, the purpose of the present investigation was to assess the interactions of CLA and R-ALA (at low, individually minimally effective doses, and at high, individually maximally effective doses) on whole-body glucose tolerance and insulin-stimulated glucose transport in skeletal muscle in an animal model of insulin resistance-the female obese Zucker rat. In addition, the roles of reductions in oxidative stress and muscle lipid storage in this interaction were assessed.

From the Muscle Metabolism Laboratory, Department of Physiol ogy, University of Arizona College of Medicine, Tucson, AZ; AlbertSchweitzer-Klinik, Königsfeld, Germany; and BASF AG, Ludwigshafen, Germany.

Submitted December 7, 2002; accepted February 23, 2003.

Supported by a grant from BASF AG, Ludwigshafen, Germany, to E.J.H.

Address reprint requests to Erik J. Henriksen, PhD, Department of Physiology, Ina E. Gittings Building \#93, University of Arizona, Tucson, AZ 85721-0093.

(C) 2003 Elsevier Inc. All rights reserved.

0026-0495/03/5209-0042\$30.00/0

doi:10.1016/S0026-0495(03)00145-8 


\section{MATERIALS AND METHODS}

\section{Animals and Treatments}

All procedures described below were approved by the University of Arizona Animal Care and Use Committee. Female obese Zucker rats (Hsd/Ola:ZUCKER-fa; Harlan, Indianapolis, IN) were received at 6 to 7 weeks of age. They were housed in a temperature controlled room (20 to $22^{\circ} \mathrm{C}$ ) at the Central Animal Facility of the University of Arizona, maintained on a 12:12-hour light-dark cycle, and had free access to water and standard chow (Teklad 7001 4\% Mouse/Rat Diet) (Teklad, Madison, WI) throughout the study. Starting at 8 to 9 weeks of age, animals received for 20 consecutive days either vehicle (corn oil by gavage and $100 \mathrm{mmol} / \mathrm{L}$ Tris [pH 7.4] intraperitoneally [IP]), a 50:50 mixture of cis-9,trans- 11 and trans-10,cis- 12 isomers of CLA $(0.3 \mathrm{~g} / \mathrm{kg}$ or $1.5 \mathrm{~g} / \mathrm{kg}$ body weight) daily by gavage, the R-ALA $(10 \mathrm{mg} / \mathrm{kg}$ or 50 $\mathrm{mg} / \mathrm{kg}$ IP), or combinations of the low doses of CLA and R-ALA or of the high doses of CLA and R-CLA in combination.

\section{Oral Glucose Tolerance Tests}

After 18 days of treatment, animals were fasted (food was restricted to $4 \mathrm{~g}$ of chow at $5 \mathrm{pm}$ the evening before the test, and was consumed within 1 to 2 hours). Between 8 am and 10 am, approximately 12 hours after the most recent treatment, the animals underwent an oral glucose tolerance test (OGTT) using a $1-\mathrm{g} / \mathrm{kg}$ body weight glucose feeding by gavage. Blood $(0.1 \mathrm{~mL})$ was collected from a cut at the tip of the tail immediately before and 30, 60, 90, and 120 minutes following the glucose feeding, and was thoroughly mixed with EDTA $(18 \mathrm{mmol} / \mathrm{L}$ final concentration) and then centrifuged at $13,000 \times g$ to separate the plasma. The plasma was stored at $-80^{\circ} \mathrm{C}$ until analyzed for glucose (Sigma, St Louis, MO), insulin (Linco Research, St Charles, MO), and free fatty acids (FFA) (Wako, Richmond, VA). Immediately after the completion of the OGTT, all animals received $2.5 \mathrm{~mL}$ of sterile $0.9 \%$ saline subcutaneously to compensate for plasma loss. Treatments resumed for 2 further days.

\section{Glucose Transport Activity}

On the evening of day 20, animals were food-restricted as described above. Between 8 am and 10 am, approximately 12 hours after the final treatments, animals were deeply anesthetized with pentobarbital sodium $(50 \mathrm{mg} / \mathrm{kg} \mathrm{IP})$. Both epitrochlearis muscles and 1 soleus muscle were surgically removed and prepared for in vitro incubation. Epitrochlearis muscles were incubated intact, while soleus muscles were prepared into strips (weighing about $25 \mathrm{mg}$ ). ${ }^{24}$ The muscles were initially incubated (without tension throughout) for 60 minutes in $3 \mathrm{~mL}$ of oxygenated Krebs-Henseleit buffer (KHB) containing $8 \mathrm{mmol} / \mathrm{L}$ glucose, $32 \mathrm{mmol} / \mathrm{L}$ mannitol, and $0.1 \%$ bovine serum albumin (BSA, radioimmunoassay grade). One muscle preparation from each animal was incubated in the absence of insulin, while the contralateral muscle preparation was incubated in medium containing $2 \mathrm{mU} / \mathrm{mL}$ of insulin (Humulin, Eli Lilly, Indianapolis, IN). The flasks were shaken in a Dubnoff incubator at $37^{\circ} \mathrm{C}$ and had a gas phase of $95 \% \mathrm{O}_{2}: 5 \% \mathrm{CO}_{2}$. Following the initial treatments, all muscles were rinsed for 10 minutes at $37^{\circ} \mathrm{C}$ in $3 \mathrm{~mL}$ of oxygenated $\mathrm{KHB}$ containing $40 \mathrm{mmol} / \mathrm{L}$ mannitol, $0.1 \% \mathrm{BSA}$, and, if present previously, insulin. The muscles were then transferred to flasks containing $2 \mathrm{~mL}$ of oxygenated KHB, $0.1 \%$ BSA, $1 \mathrm{mmol} / \mathrm{L} 2$-deoxy $\left[1,2-{ }^{3} \mathrm{H}\right]$ glucose (2-DG) $(300 \mu \mathrm{Ci} / \mathrm{mmol}$; Sigma), $39 \mathrm{mmol} / \mathrm{L}\left[\mathrm{U}-{ }^{14} \mathrm{C}\right] \mathrm{mannitol}(0.8 \mu \mathrm{Ci} / \mathrm{mmol})$ (ICN Radiochemicals, Irvine, CA), and insulin, if present previously. After this final 20minute incubation period at $37^{\circ} \mathrm{C}$, muscles were trimmed of fat, extraneous muscle, and connective tissue, frozen between aluminum blocks cooled to the temperature of liquid $\mathrm{N}_{2}$, weighed, and dissolved in 0.5 $\mathrm{mL}$ of $0.5 \mathrm{~N} \mathrm{NaOH}$. After complete solubilization, $5 \mathrm{~mL}$ of scintillant was added and samples were analyzed for radioactivity in the ${ }^{3} \mathrm{H}$ and ${ }^{14} \mathrm{C}$ channels. The radioactivity in the ${ }^{14} \mathrm{C}$ channel and the specific activity of the incubation medium were used to determine the extracellular space, while the specific uptake of 2DG was calculated by subtracting the ${ }^{3} \mathrm{H}$ activity in the extracellular space from the total ${ }^{3} \mathrm{H}$ activity in each sample. This method for assessing glucose transport activity in isolated muscle has been validated. ${ }^{25}$

\section{Biochemical Assays}

At the time of muscle dissection, the second soleus muscle was removed and frozen in liquid nitrogen. Frozen sections of this muscle (30 to $50 \mathrm{mg}$ ) were used for the measurement of protein carbonyl levels, using the spectrophotometric assay of Reznick and Packer, ${ }^{26}$ and the assessment of muscular triglyceride concentration, using the chloroform-methanol extraction described by Folch et al, ${ }^{27}$ followed by the processing method of Frayn and Maycock, ${ }^{28}$ as modified by Denton and Randle. ${ }^{29}$ Glycerol was ultimately assayed spectrophotometrically using a commercially available kit (Sigma Chemical).

\section{Statistical Analysis}

All data are presented as means \pm SE. Significant differences between multiple groups within either the low-dose-treated animals or the high-dose-treated animals were assessed by analysis of variance (ANOVA) with a post hoc Fisher protected least significant difference (PLSD) test (Statview II, Abacus Concepts, Piscataway, NJ). Correlations were analyzed by simple linear regression. Statistical significance was set at the .05 probability level.

\section{RESULTS}

\section{Low-Dose Treatments}

The final body weights of the obese Zucker rats $(n=5$ or 6 animals per group) in the low-dose treatment groups were not different from the vehicle-treated group (Table 1). Mean beginning body weights of the low-dose groups ( $278 \pm 4 \mathrm{~g})$ were not different from those of the vehicle-treated groups (267 \pm $6 \mathrm{~g})$.

Fasting plasma values for glucose, insulin, and FFAs in the low-dose-treated obese animals are presented in Table 1. Whereas low-dose R-ALA did not have an effect on any of these variables, low-dose CLA treatment caused a 5\% decrease in plasma glucose and a $16 \%$ decrease in plasma FFA compared to the vehicle-treated group. These modest low-dose CLA-induced decreases in plasma glucose and FFA were maintained in the combination treatment group $(P<.05)$. There was a tendency for small decreases (9\% to $11 \%$ ) in plasma insulin following low-dose CLA and combination treatments, but these differences did not achieve statistical significance.

The glucose and insulin responses during the OGTT in the low-dose groups are shown in Fig 1 (upper panels). At the 30-minute time point, the glucose values in the low-dose RALA, CLA, and combination treatment groups were significantly lower than in the vehicle-treated group. At the 60- and 90-minute time points, only the glucose value in the combination treatment group was significantly less than the vehicle control value. The insulin values in the low-dose CLA and the combination treatment groups were significantly lower than the low-dose R-ALA group at the 60-minute time point. The integrated area under the curve (AUC, 0 to 120 minutes) for the glucose response during the OGTT was $6 \%$ less than vehicle control in the CLA group and $11 \%$ less than vehicle control in the combination treatment group (Fig 2, upper panels). There 
Table 1. Effects of Low-Dose or High-Dose CLA and R-ALA Treatments on Fasting Plasma Variables in Female Obese Zucker Rats

\begin{tabular}{|c|c|c|c|c|}
\hline Group & $\begin{array}{l}\text { Final Body } \\
\text { Weights (g) }\end{array}$ & $\begin{array}{l}\text { Plasma Glucose } \\
(\mathrm{mg} / \mathrm{dL})\end{array}$ & $\begin{array}{l}\text { Plasma Insulin } \\
\qquad(\mu \mathrm{U} / \mathrm{mL})\end{array}$ & $\begin{array}{c}\text { Plasma FFA } \\
(\mathrm{mmol} / \mathrm{L})\end{array}$ \\
\hline \multicolumn{5}{|c|}{ Low-dose treatments } \\
\hline Vehicle-treated & $315 \pm 7$ & $119 \pm 1$ & $160 \pm 11$ & $1.64 \pm 0.04$ \\
\hline $10 \mathrm{mg} / \mathrm{kg} \mathrm{R}-\mathrm{ALA}$ & $326 \pm 3$ & $117 \pm 1$ & $154 \pm 14$ & $1.55 \pm 0.07$ \\
\hline $0.3 \mathrm{~g} / \mathrm{kg} \mathrm{CLA}$ & $309 \pm 4$ & $114 \pm 2^{*}$ & $143 \pm 4$ & $1.37 \pm 0.07^{*} \dagger$ \\
\hline Combined & $328 \pm 4$ & $113 \pm 2^{*}$ & $145 \pm 5$ & $1.37 \pm 0.05^{*} \dagger$ \\
\hline \multicolumn{5}{|c|}{ High-dose treatments } \\
\hline Vehicle-treated & $334 \pm 6$ & $118 \pm 3$ & $163 \pm 7$ & $1.71 \pm 0.02$ \\
\hline $50 \mathrm{mg} / \mathrm{kg}$ R-ALA & $313 \pm 3^{*}$ & $115 \pm 2$ & $151 \pm 7$ & $1.44 \pm 0.04^{*}$ \\
\hline $1.5 \mathrm{~g} / \mathrm{kg} \mathrm{CLA}$ & $313 \pm 5^{*}$ & $113 \pm 3$ & $124 \pm 6 * \dagger$ & $1.39 \pm 0.03^{*}$ \\
\hline Combined & $318 \pm 8$ & $115 \pm 3$ & $137 \pm 9 *$ & $1.31 \pm 0.04^{*} \dagger$ \\
\hline
\end{tabular}

NOTE. Values are means \pm SE for 6 animals in the vehicle-treated groups and 5 animals in all other groups.

${ }^{*} P<.05 v$. respective vehicle-treated group.

† $P<.05 v$. respective R-ALA group.

were no significant differences due to the treatments for the insulin AUC.

The glucose-insulin index, defined as the product of the glucose AUC and the insulin AUC and inversely related to whole-body insulin sensitivity, ${ }^{15,19}$ was significantly less in the low-dose CLA-treated group (17\%) and in the combination treatment group (21\%) compared to the vehicle-treated group (Fig 2, upper right panel). In addition, the glucose-insulin index was significantly less $(16 \%)$ in the combination treatment group compared to the R-ALA group.

To assess whether these alterations in whole-body glucose disposal were associated with changes in the skeletal muscle glucose transport system, insulin-mediated 2-DG uptake in isolated skeletal muscles was determined (Fig 3, upper panels). Low-dose R-ALA had no effect on this variable. In contrast, low-dose CLA treatment increased (38\%) insulin- mediated glucose transport activity in the epitrochlearis, but not in the soleus. The combination treatment caused the greatest enhancement of insulin-mediated glucose transport activity in both the epitrochlearis (77\%) and the soleus (54\%).

Oxidative stress may play a role in the development of insulin resistance, ${ }^{30,31}$ and the level of carbonyl formation in proteins is an indicator of oxidative damage in tissues, ${ }^{32}$ reflecting the degree of oxidative stress. ${ }^{26}$ Low-dose treatments with R-ALA or CLA individually did not alter the elevated level of protein carbonyls in the soleus of the obese Zucker rat (Fig 4). However, combination treatment with low doses of these compounds was associated with a $50 \%$ reduction in protein carbonyls in the soleus.

Soleus muscle triglycerides were likewise not altered by the individual low-dose treatments with R-ALA or CLA, but were reduced by $33 \%$ in the low-dose combination treatment
Fig 1. Glucose and insulin responses during an OGTT in female obese Zucker rats after lowdose (upper panels) or after highdose (lower panels) R-ALA, CLA, or combination treatments. Values are means \pm SE for 5 or 6 animals per group. Errors bars not shown were either smaller than the size of the symbol or were omitted to enhance clarity of the figure. ${ }^{a} P<.05 v$ vehicletreated group. ${ }^{\mathrm{b}} \boldsymbol{P}<.05 \mathrm{v}$ lowdose R-ALA.
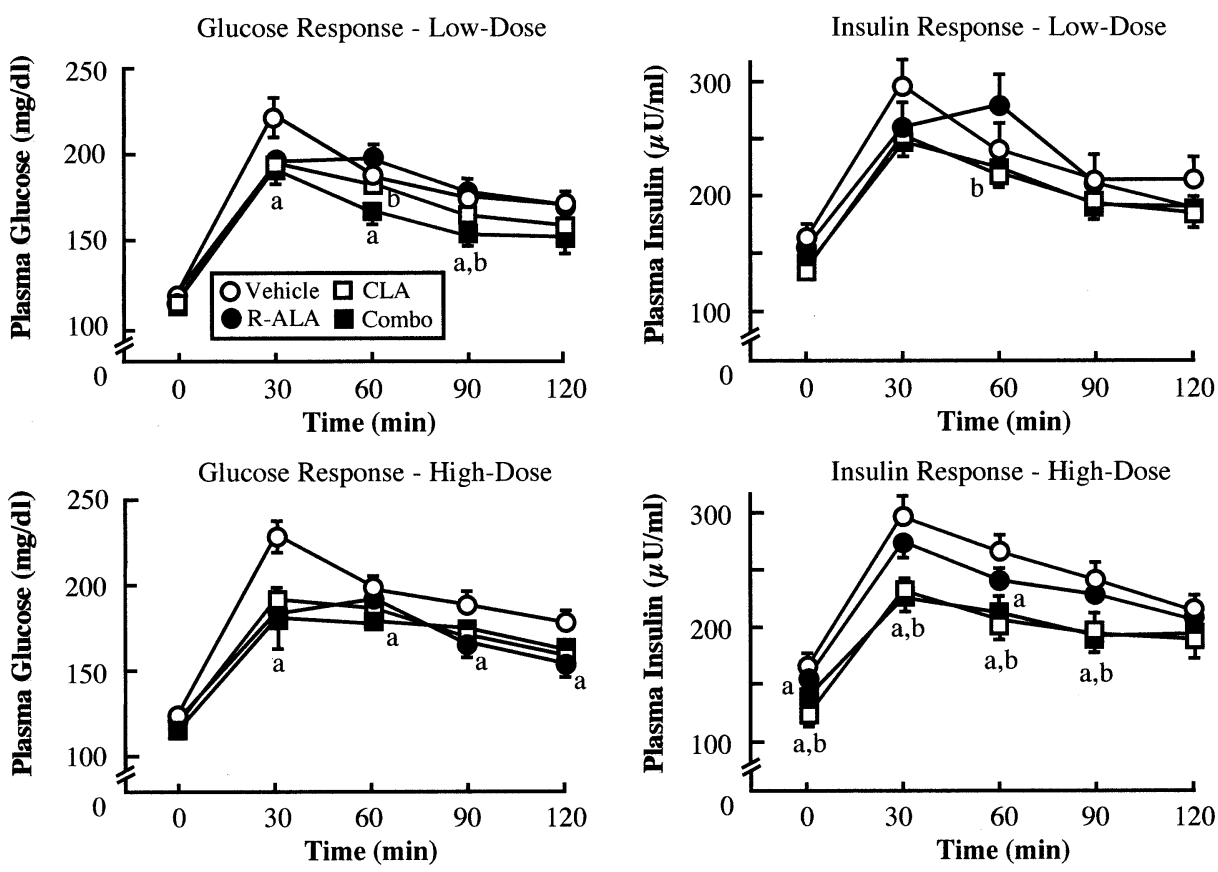

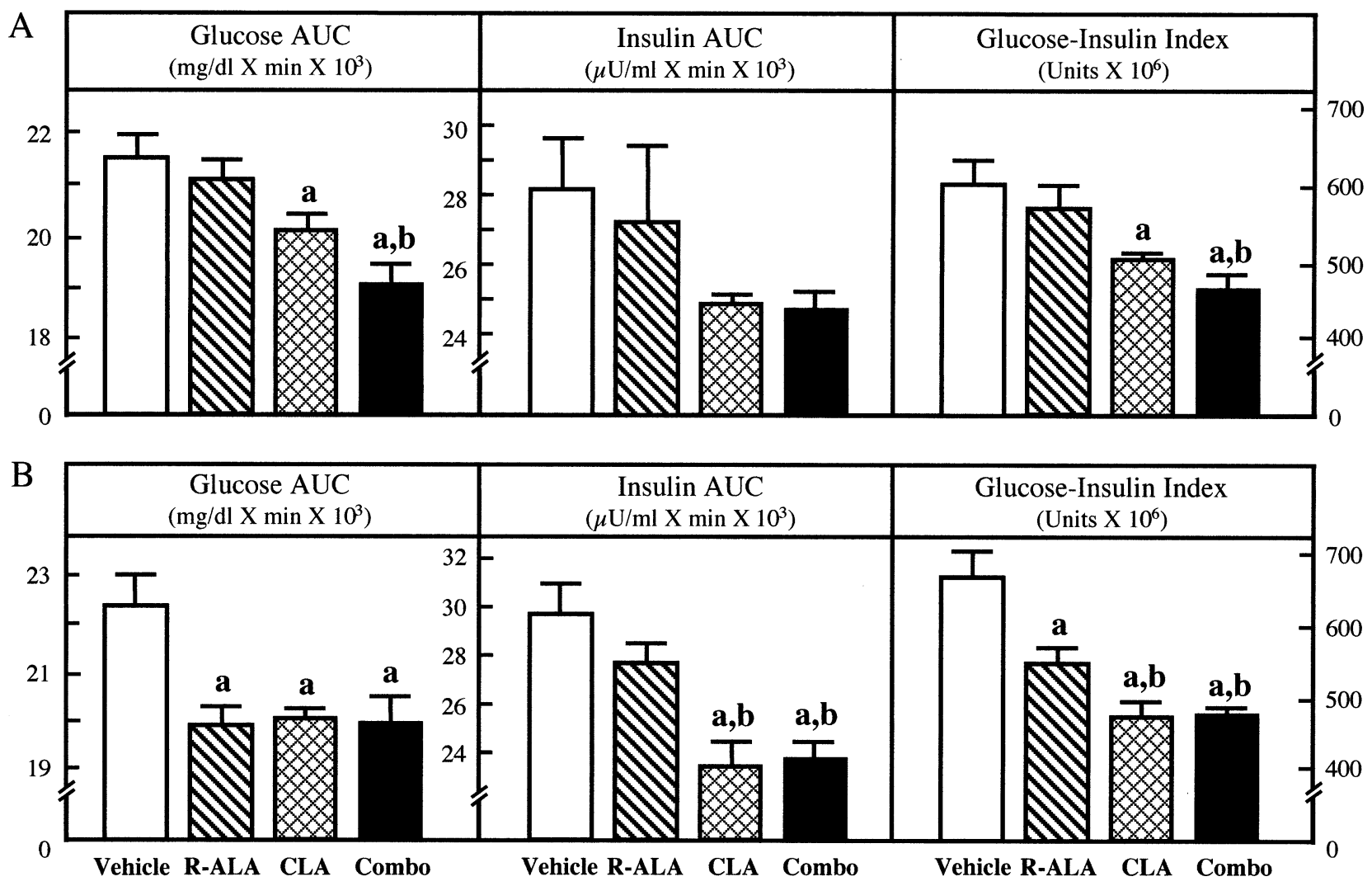

Fig 2. Areas under the curves for glucose and insulin during the oral glucose tolerance tests and the glucose-insulin index in female obese Zucker rats after the respective low-dose (part A, upper panels) and high-dose (part B, lower panels) treatments. Values are means \pm SE for 5 or 6 animals per group. Data were taken from Fig 1 . ${ }^{\text {a } P}<.05 v$ vehicle-treated group. ${ }^{b} P<.05 v$ the respective $R$-ALA group.

group compared to the vehicle control group (Fig 5, upper panel).

\section{High-Dose Treatments}

The final body weights ( $\mathrm{n}=5$ to 6 animals per group) in the high-dose R-ALA and high-dose CLA groups, but not in the high-dose R-ALA/CLA combination group, were significantly less $(P<.05)$ than that of the vehicle-treated group (Table 1). The initial body weights of the high-dose groups $(280 \pm 3 \mathrm{~g})$ were not different from those of the vehicle-treated group $(278 \pm 6 \mathrm{~g})$.

The values for fasting plasma glucose, insulin, and FFAs in the high-dose groups are displayed in Table 1 . None of the high-dose interventions significantly altered plasma glucose. However, high-dose CLA treatment brought about a $24 \%$ decrease in plasma insulin, and this reduction in plasma insulin was essentially maintained in the high-dose combination treatment. There was a $16 \%$ decline in plasma FFA following treatment with high-dose R-ALA. Likewise, high-dose CLA treatment induced a $19 \%$ decrease in plasma FFA, and a $23 \%$ reduction in plasma FFA levels was observed in the high-dose combination treatment group.

During the OGTT, the glucose values in the high-dose RALA $(21 \%)$, CLA (18\%), and combination treatment $(21 \%)$ groups were significantly less than the vehicle control value at the 30-minute time point (Fig 1, lower panels). At the 60- minute time point, the combination treatment group value was $10 \%$ less than control, while at the at the 90- and 120-minute time points, only the R-ALA group was significantly less (11\% and $10 \%$, respectively) than control. The insulin values during the OGTT were significantly less than control in the high-dose CLA and combination treatment groups at the 30-minute (23\% and $24 \%$ ), 60-minute (23\% and $19 \%$ ), and 90 -minute (21\% and $21 \%$ ) time points. The glucose AUCs during the OGTT were $11 \%, 10 \%$, and $11 \%$ less than vehicle control in the high-dose R-ALAtreated, CLA-treated, and combination treatment groups, respectively. The insulin AUC was significantly less than the vehicletreated control value in the high-dose CLA-treated group (21\%) and in the combination treatment group (20\%).

The glucose-insulin index was $17 \%$ less than vehicle-treated control in the high-dose R-ALA treatment groups, $29 \%$ less than control in the high-dose CLA group, and $28 \%$ less than control in the combination treatment group (Fig 2, lower right panel).

High-dose R-ALA treatment alone induced increases of $58 \%$ and $35 \%$ in insulin-mediated glucose transport activity the epitrochlearis and soleus, respectively. The high-dose CLA treatment was also associated with significant increases in this variable in the epitrochlearis $(57 \%)$ and in the soleus $(32 \%)$ of the obese Zucker rat. Moreover, the high-dose combination treatment caused the greatest enhancement of insulin-mediated 


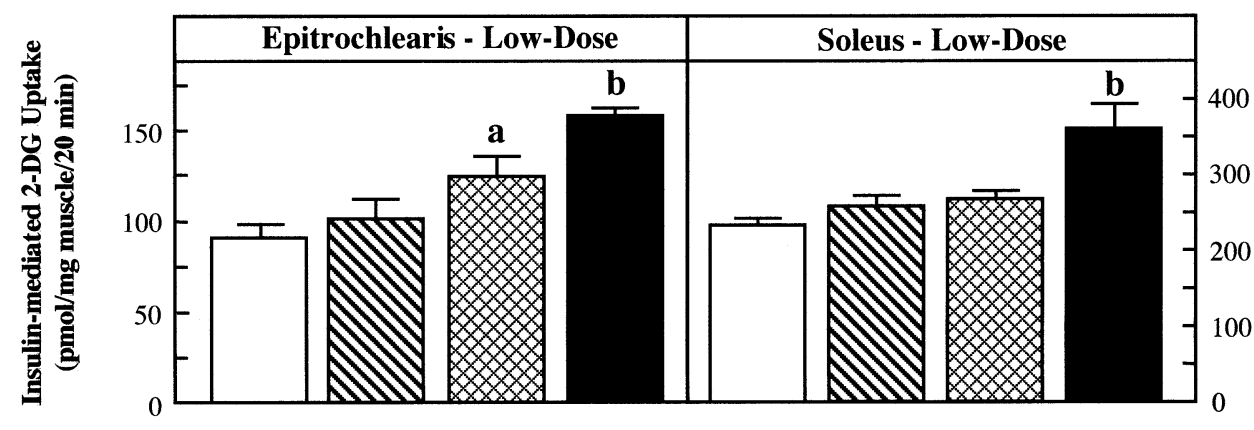

Fig 3. Interactions of R-ALA and CLA on insulin-mediated glucose transport activity in the epitrochlearis and soleus muscles of female obese Zucker rats. Values for the low-dose treatment groups are in the upper panels and values for the highdose groups are in the lowe panels. Values are means $\pm S E$ for $\mathbf{5}$ or $\mathbf{6}$ animals per group. ${ }^{\text {a }} \boldsymbol{P}$ $<.05 v$ vehicle-treated group ${ }^{b} P<.05 v$ all other groups within that treatment regimen.

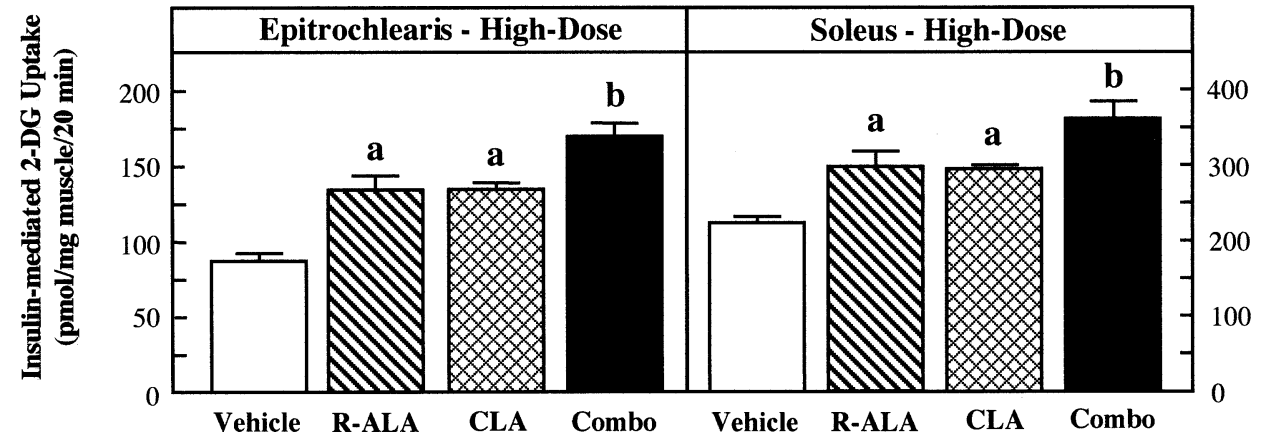

glucose transport activity in both the epitrochlearis (77\%) and the soleus $(54 \%)$

High-dose treatments with R-ALA or CLA individually lowered protein carbonyl levels in the soleus of the obese Zucker
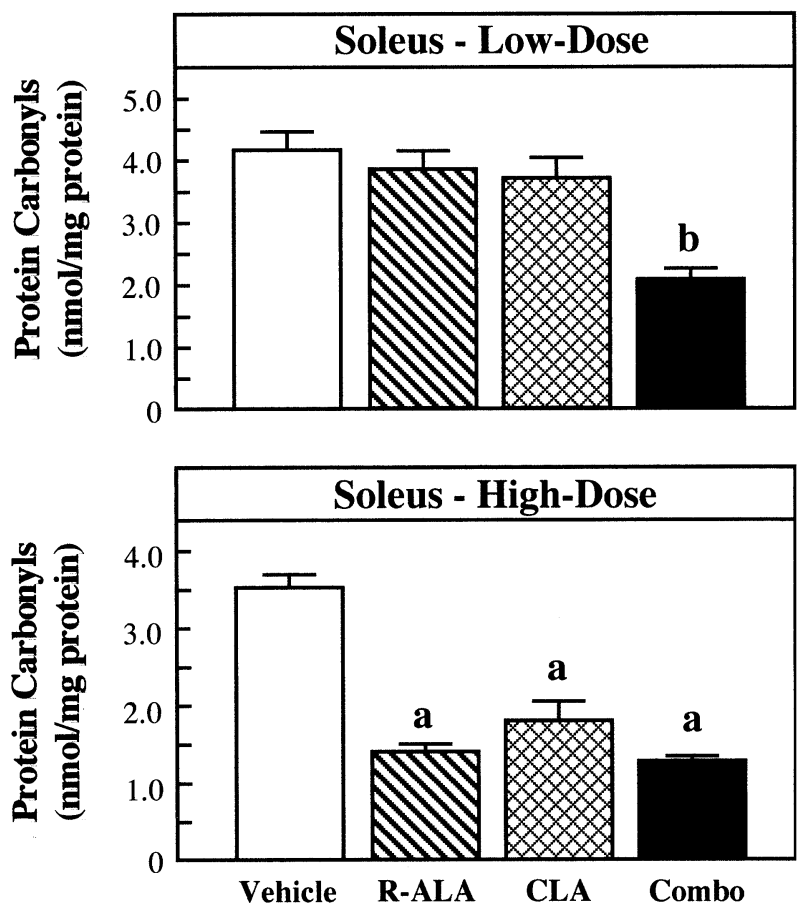

Fig 4. Effects of low-dose or high-dose R-ALA and CLA treatments on protein carbonyl levels in soleus muscle of female obese Zucker rats. Values are means \pm SE for 5 or 6 animals per group. ${ }^{a} P<.05 v$ vehicle-treated group. ${ }^{b} P<.05 v$ all other groups. rat by $63 \%$ and $49 \%$, respectively (Fig 4, lower panel). This reduction $(64 \%)$ in protein carbonyl level was maintained in the soleus muscle of obese Zucker rats that received the high-dose combination treatment.

Soleus muscle triglycerides were reduced $29 \%$ and $28 \%$, respectively, by the individual high-dose treatments with $\mathrm{R}$ ALA or CLA, and were reduced only slightly more (39\%) in the high-dose combination treatment group compared to the vehicle control group (Fig 5, lower panel).

\section{Correlational Analysis}

To quantitatively assess the association between insulinmediated glucose transport activity in the soleus muscle of the obese Zucker rats and muscle variables known to affect insulin action, correlational analyses were conducted (Fig 6). Significant inverse correlations were observed in the soleus from the low-dose-treated animals between insulin-mediated glucose transport and protein carbonyls (Fig 6A, $r=-0.617$ ) and between insulin-mediated glucose transport and intramuscular triglycerides (Fig 6B, $r=-0.515$ ). In addition, significant inverse correlations were observed in the soleus from the high-dose-treated animals between insulin-mediated glucose transport and protein carbonyls (Fig 6C, $r=-0.761$ ) and between insulin-mediated glucose transport and intramuscular triglycerides (Fig 6D, $r=-0.625$ ).

\section{DISCUSSION}

In this investigation, a novel interaction between the fatty acid CLA and the antioxidant R-ALA in the modulation of whole-body and skeletal muscle insulin resistance in the female obese Zucker rat has been described. Whereas the individual chronic effects of CLA and R-ALA at low doses were relatively small $(0.3 \mathrm{~g} / \mathrm{kg}$ CLA) or undetectable $(10 \mathrm{mg} / \mathrm{kg} \mathrm{R}$ ALA), when these compounds were provided in combination 

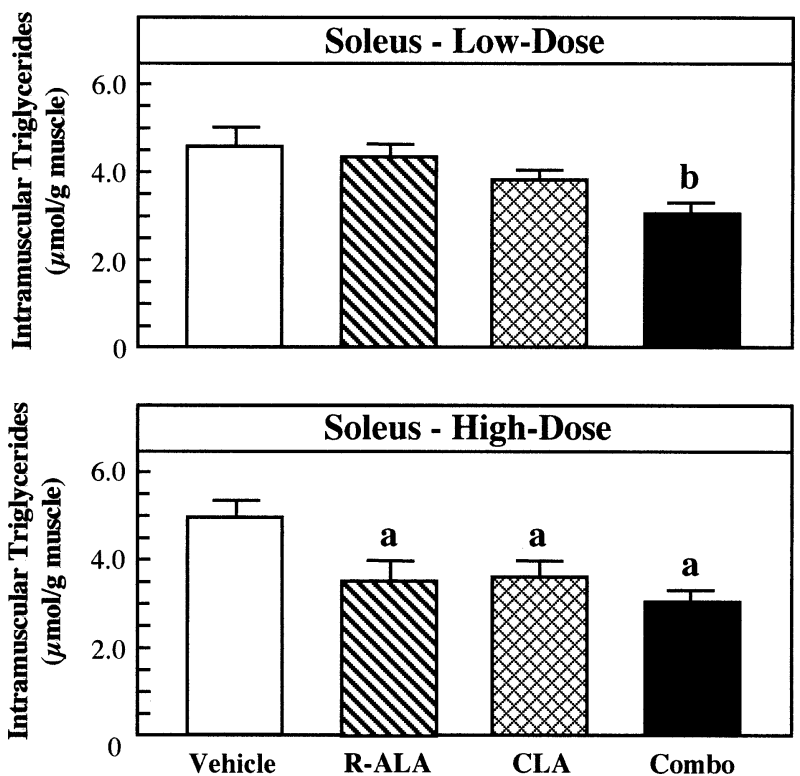

Fig 5. Effects of low-dose or high-dose R-ALA and CLA treatments on triglyceride levels in soleus muscle of female obese Zucker rats. Values are means \pm SE for 5 or 6 animals per group. ${ }^{\text {a } P}<.05 v$ vehicle-treated group. ${ }^{b} P<.05 v$ all other groups.

chronically to the obese Zucker rat, there were substantial and significant improvements in whole-body glucose tolerance and insulin sensitivity (Figs 1 and 2) and in insulin-mediated glucose transport activity in type I (soleus) and type IIb (epitrochlearis) skeletal muscle (Fig 3). Interestingly, at high doses of CLA and R-ALA (1.5 g/kg and $50 \mathrm{mg} / \mathrm{kg})$, which by them- selves elicited significant enhancements of whole-body and skeletal muscle insulin action, the interactions between the 2 compounds were much less evident (Figs 1 to 3 ).

Oxidative stress, the consequence of a dysequilibrium between the production of pro-oxidants and the various antioxidant defenses in plasma and tissues, can be linked to the development or worsening of skeletal muscle insulin resistance. ${ }^{30,31}$ It has been shown recently that enhanced formation of protein carbonyls in muscle and liver, one indicator of oxidative stress-associated tissue damage, ${ }^{26,32}$ is associated with whole-body and skeletal muscle insulin resistance in the female obese Zucker rat. ${ }^{15}$ Moreover, chronic treatment of these animals with R-ALA reduces tissue protein carbonyl levels and improves insulin-mediated skeletal muscle glucose transport activity. ${ }^{15}$ Leung and $\mathrm{Liu}^{13}$ have demonstrated that CLA, especially the trans-10, cis-12 isomer, can act in vitro as a scavenger of reactive oxygen species. In the present investigation, low doses of CLA and R-ALA were shown to interact to elicit a greater reduction in protein carbonyls in soleus muscle of the obese Zucker rat than either treatment individually (Fig 4), and this reduction in oxidative stress significantly correlated with the observed enhancement of insulin-mediated glucose transport activity (Fig 6A). While the high doses of CLA and R-ALA also reduced muscle oxidative stress (Fig 3), it is clear that the combination of the high doses of CLA and R-ALA did not elicit any further reductions in this parameter. These data are consistent with the concept that the beneficial metabolic interactions between CLA and R-ALA, especially at the lower doses, may be attributed, at least in part, to their antioxidant properties.

There is generally an inverse relationship between the concentration of intramuscular lipid and insulin-dependent glucose
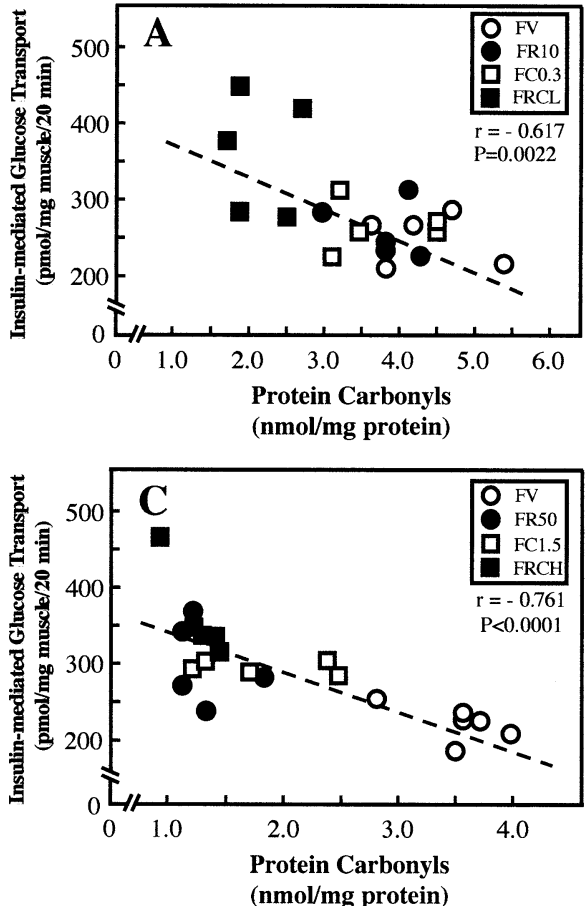

Fig 6. Correlations between insulin-mediated glucose transport activity and either protein carbonyl level (left panels) or triglyceride level (right panels) in soleus muscle of female obese Zucker rats treated with lowdose (upper panels) or high-dose (lower panels) R-ALA and CLA. Data were taken from Figs 3 through 5. FV, vehicle-treated; FR, R-ALA-treated, FC, CLAtreated; FRCL, low-dose combination treatment; $\mathrm{FRCH}$, highdose combination treatment. 
transport in sedenatry rodent or human muscle, ${ }^{33-35}$ possibly due to a disruption of insulin signaling by fatty acid moieties, such long-chain fatty acyl coenzyme A $(\mathrm{CoA}) .{ }^{36}$ It has been observed that triglyceride levels are 3-fold higher in insulinresistant soleus muscle of the female obese Zucker rat compared to those levels in soleus muscle of the insulin-sensitive female lean Zucker rat. ${ }^{37}$ In the present study, it was shown that while the low doses of CLA and R-ALA individually did not cause a decrease in intramuscular triglycerides in the soleus muscle of the obese Zucker rat, the combination of these 2 low-dose interventions elicited a substantial diminution in muscle lipids (Fig 5) that was significantly correlated with the enhancement of insulin-mediated glucose transport activity in these muscles (Fig 6B). High doses of CLA and R-ALA individually also reduced soleus muscle triglycerides (Fig 5), but the high-dose combination treatment did not significantly decrease muscle triglycerides any further. It is clear that the significant correlation between muscle triglycerides and insulin-mediated glucose transport activity in the high-dose groups was primarily due to effects of the individual treatments (Fig 6D). Collectively, these data support the hypothesis that the reduction in muscle triglycerides contributes to the beneficial metabolic interactions between CLA and R-ALA, primarily in the low-dose treatment groups.

It is well established that elevated plasma FFAs can negatively affect insulin action, ${ }^{38}$ and fasting plasma FFAs are markedly increased in the female obese Zucker rat (Table $1)^{15,17-19}$ compared to the female lean Zucker rat. ${ }^{16-18}$ In the present investigation, reductions in plasma FFAs due to the interventions were in most cases accompanied by improvements in whole-body insulin sensitivity. Low-dose CLA treatment and high-dose treatments with R-ALA or CLA significantly decreased fasting plasma FFA levels (Table 1) and improved glucose tolerance and insulin sensitivity (Fig 2), possibly a consequence of the diminution of the negative impact of the FFAs on muscle glucose disposal. However, it should be emphasized that the interactions between CLA and R-ALA in the low-dose and high-dose combination treatment groups were not attributable to further alterations in plasma FFA levels, as the combination treatments did not lower FFA levels to a greater degree than the individual treatments with CLA (Table 1).

To our knowledge, all investigations of the metabolic actions of the mixed isomers of CLA in the context of insulin resistance have utilized high doses (usually $1.5 \mathrm{~g} / \mathrm{kg}$ body weight). ${ }^{8} 9 \mathrm{In}$ the present investigation, some improvements in the deranged meta- bolic regulation of the female obese Zucker rat were elicited at a substantially lower dose of CLA $(0.3 \mathrm{mg} / \mathrm{kg})$. For example, the low-dose CLA treatment caused decreases in fasting plasma glucose and FFAs (Table 1), improved whole-body glucose tolerance and insulin sensitivity (Fig 2), and increased insulin-mediated glucose transport activity in fast-glycolytic muscle (Fig 3). Therefore, the beneficial effects of this fatty acid can be realized at much lower doses than previously considered, at least in this animal model of insulin resistance.

A clear improvement in whole-body and skeletal muscle insulin action following chronic oral administration of CLA to the obese Zucker rat (Figs 2 and 3) was shown in the present investigation, consistent with previous investigations in rat models of severe obesity-associated insulin resistance. ${ }^{8,9}$ These CLA-induced improvements in glucose metabolism were possibly related to a decrease in muscle oxidative stress (Figs 4 and 6). However, recent studies by Riserus et al ${ }^{39,40}$ in abdominally obese human males have indicated that treatment with CLA (specifically the trans-10, cis-12 isomer) actually worsened slightly insulin resistance (19\%) and glycemia (4\%) and was associated with an increase in oxidative stress (based on plasma isoprostanes). Based on these limited clinical findings, and in the context of the positive results from the present study and previous investigations, ${ }^{8,9}$ it is clear that subjects in human clinical trials of CLA must be selected very carefully to ensure that they will be appropriate candidates for CLA treatment of obesity and insulin resistance.

In conclusion, the present study describes a novel interaction between chronic treatments with the fatty acid CLA and the antioxidant R-ALA for the enhancement of glucose tolerance and insulin action on skeletal muscle glucose transport in the markedly insulin-resistant, glucose-intolerant, hyperinsulinemic, and dyslipidemic female obese Zucker rat. At low doses of these compounds, the synergistic interaction on improvement of insulin-mediated glucose transport in muscle is associated with reductions in both muscle oxidative stress and intramuscular triglycerides. At high doses of CLA and R-ALA, which by themselves substantially improve the glucose tolerance and skeletal muscle insulin action, these interactions were less evident. It is clear that further investigation is necessary to elucidate the mechanism underlying these interactions. Nevertheless, these findings support the possible use of combined CLA and R-ALA as an intervention to improve insulin action in conditions of insulin resistance and glucose intolerance associated with central obesity, hyperinsulinemia, and dyslipidemia.

\section{REFERENCES}

1. Shepherd PR, Kahn BB: Glucose transporters and insulin action. N Engl J Med 341:248-257, 1999

2. Zierath JR, Krook A, Wallberg-Henriksson H: Insulin action and insulin resistance in human skeletal muscle. Diabetologia 43:821-835, 2000

3. Reaven GM: Role of insulin resistance in human disease (syndrome X): An expanded definition. Annu Rev Med 44:121-131, 1993

4. DeFronzo RA, Ferrannini E: Insulin resistance: A multifaceted syndrome responsible for NIDDM, obesity, hypertension, dyslipidemia, and atherosclerotic cardiovascular disease. Diabetes Care 14:173-194, 1991

5. Henriksen EJ: Invited review: Effects of acute exercise and exercise training of insulin resistance. J Appl Physiol 93:788-796, 2002
6. Ip C, Scimeca JA, Thompson HJ: Conjugated linoleic acid. A powerful anticarcinogen from animal fat stores. Cancer 74:1050-1054, 1994 (suppl)

7. Blankson H, Stakkestad JA, Fagertun H, et al: Conjugated linoleic acid reduces body fat mass in overweight and obese humans. J Nutr 130:2943-2948, 2000

8. Houseknecht KL, Vanden Heuvel JP, Moya-Camarena SY, et al: Dietary conjugated linoleic acid normalizes impaired glucose tolerance in the Zucker Diabetic Fatty fa/fa rat. Biochem Biophys Res Commun 244:678-682, 1998

9. Ryder JW, Portocarrero CP, Song XM, et al: Isomer-specific antidiabetic properties of conjugated linoleic acid. Improved glucose 
tolerance, skeletal muscle insulin action, and UCP-2 gene expression. Diabetes 50:1149-1157, 2001

10. Park Y, Albright KJ, Lui W, et al: Effect of conjugated linoleic acid on body composition in mice. Lipids 32:853-858, 1997

11. Park Y, Storkson JM, Albright KJ, et al: Evidence that the trans-10, cis-12 isomer of conjugated linoleic acid induces body composition changes in mice. Lipids 34:235-241, 1999

12. Delaney JP, Blohm F, Truett AA, et al: Conjugated linoleic acid rapidly reduces body fat content in mice without affecting energy intake. Am J Physiol Regul Int Comp Physiol 276:R1172-R1179, 1999

13. Leung YH, Liu RH: Trans-10, cis-12-conjugated linoleic acid isomer exhibits stronger oxyradical scavenging capacity than cis-9, trans-11-conjugated linoleic acid isomer. J Agricult Food Chem 48: $5469-5475,2000$

14. Kraemer K, Packer L: R- $\alpha$-Lipoic acid, in Kraemer K, Hoppe PP, Packer L (eds): Nutriceuticals in Health and Disease Prevention. New York, NY, Dekker, 2001, pp 129-163

15. Saengsirisuwan V, Kinnick TR, Schmit MB, et al: Interactions of exercise training and alpha-lipoic acid on glucose transport in obese Zucker rat. J Appl Physiol 91:145-153, 2001

16. Saengsirisuwan V, Perez FR, Kinnick TR, Henriksen EJ: Effects of exercise training and antioxidant R-ALA on glucose transport in insulin-sensitive rat skeletal muscle. J Appl Physiol 92:50-58, 2002

17. Jacob S, Streeper RS, Fogt DL, et al: The antioxidant alphalipoic acid enhances insulin stimulated glucose metabolism in insulinresistant rat skeletal muscle. Diabetes 45:1024-1029, 1996

18. Streeper RS, Henriksen EJ, Jacob S, et al: Differential effects of lipoic acid stereoisomers on glucose metabolism in insulin-resistant skeletal muscle. Am J Physiol Endocrinol Metab 273:E185-E191, 1997

19. Peth JA, Kinnick TR, Youngblood EB, et al: Effects of a unique conjugate of alpha-lipoic acid and gamma-linoleic acid on insulin action in the obese Zucker rat. Am J Physiol Regul Int Comp Physiol 279:R453-R459, 2000

20. Jacob S, Henriksen EJ, Schiemann AL, et al: Enhancement of glucose disposal in patients with type 2 diabetes by alpha-lipoic acid. Drug Res 45:872-874, 1995

21. Jacob S, Henriksen EJ, Tritschler HJ, et al: Improvement of insulin stimulated glucose disposal in type 2 diabetes after repeated parenteral administration of thioctic acid. Exp Clin Endocrinol Diabetes 104:284-288, 1996

22. Jacob S, Ruus P, Hermann R, et al: Oral administration of $r a c$ - $\alpha$-lipoic acid modulates insulin sensitivity in patients with type 2 diabetes mellitus-A placebo controlled pilot trial. Free Radic Biol Med 27:309-314, 1999

23. Konrad T, Vicini P, Kusterer K, et al: alpha-Lipoic acid treatment decreases serum lactate and pyruvate concentrations and improves glucose effectiveness in lean and obese patients with type 2 diabetes. Diabetes Care 22:280-287, 1999

24. Henriksen EJ, Ritter LS: Effect of soleus unweighting on stimulation of insulin-independent glucose transport activity. J Appl Physiol 74:1653-1657, 1993
25. Hansen PA, Gulve ES, Holloszy JO: Suitability of 2-deoxyglucose for in vitro measurement of glucose transport activity in skeletal muscle. J Appl Physiol 76:979-985, 1994

26. Reznick AZ, Packer L: Oxidative damage to proteins: Spectrophotometric method for carbonyl assay. Methods Enzymol 233:357363, 1994

27. Folch J, Lees M, Sloane Stanley JH: A simple method for the isolation and purification of total lipides from animal tissues. J Biol Chem 226:497-509, 1957

28. Frayn KN, Maycock PF: Skeletal muscle triacylglycerol in the rat: methods for sampling and measurement, and studies of biological variability. J Lipid Res 21:139-144, 1980

29. Denton RM, Randle PJ: Concentration of glycerides and phospholipids in rat heart and gastrocnemius muscles: Effects of alloxandiabetes and perfusion. Biochem J 104:416-422, 1967

30. Henriksen EJ: Oxidative stress and antioxidant treatment: effects on muscle glucose transport in animal models of type 1 and type 2 diabetes, in Packer L, Rösen P, Tritschler HJ, et al (eds): Antioxidants in Diabetes Management. New York, NY, Dekker, 2000, pp 303-318

31. Ceriello A: Oxidative stress and glycemic regulation. Metabolism 49:27-29, 2000

32. Davies KJ: Protein damage and degradation by oxygen radicals. I. General aspects. J Biol Chem 262:9895-9901, 1987

33. Oakes ND, Cooney GJ, Camilleri S, et al: Mechanisms of liver and muscle insulin resistance induced by chronic high-fat feeding. Diabetes 46:1768-1774, 1997

34. Koyama K, Chen G, Lee Y, et al: Tissue triglycerides, insulin resistance, and insulin production: Implications for hyperinsulinemia of obesity. Am J Physiol Endocrinol Metab 273:E708-E713, 1997

35. Jacob S, Machann J, Rett K, et al: Associated of increased intramyocellular lipid content with insulin resistance in lean nondiabetic offspring of type 2 diabetic subjects. Diabetes 48:1113-1119, 1999

36. Cooney GJ, Thompson AL, Furler SM, et al: Muscle long-chain acyl CoA esters and insulin resistance. Ann NY Acad Sci 967:196-207, 2002

37. Saengsirisuwan V, Perez FR, Maier T, et al: Exercise training and lipoic acid upregulate IRS-1 protein expression in muscle of obese Zucker rats. Med Sci Sports Exerc 34:S275, 2002 (suppl 1, abstr)

38. Boden G: Role of fatty acids in the pathogenesis of insulin resistance and NIDDM. Diabetes 46:3-10, 1997

39. Riserus U, Arner P, Brismar K, et al: Treatment with dietary trans-10, cis-12 conjugated linoleic acid causes isomer-specific insulin resistance in obese men with the metabolic syndrome. Diabetes Care 25:1516-1521, 2002

40. Riserus U, Basu S, Jovinge S, et al: Supplementation with conjugated linoleic acid causes isomer-dependent oxidative stress and elevated C-reactive protein: A potential link to fatty acid-induced insulin resistance. Circulation 106:1925-1929, 2002 